VoL. 47 (1992) [1-12]

\title{
CALCULUS RULES FOR COMBINATIONS OF ELLIPSOIDS AND APPLICATIONS
}

\section{Alberto Seeger}

We derive formulas for the Minkowaki sum, the convex hull, the interection, and the inverse sum of a finite family of ellipsoids. We show how these formulas can be used to obtain inner and outer ellipsoidal approximations of a convex polytope.

\section{INTRODUCTION}

There is a one-to-one correspondence between the set $P_{n}$ of symmetric positive semidefinite $n \times n$ matrices, and the set of ellipsoids in $\mathbf{R}^{n}$ which are bounded and centered at the origin. One can consider, for instance, the correspondence

$$
A \in P_{n} \longmapsto E(A):=\left\{z \in \mathbf{R}^{n}:\langle z, x\rangle \leqslant[(x, A x)]^{1 / 2} \text { for all } x \in \mathbf{R}^{n}\right\} \text {, }
$$

where $\langle.,$.$\rangle stands for the usual inner product in \mathbf{R}^{n}$. The set $E(A)$ is referred to as the ellipsoid associated with $A \in P_{n}$. Note that this set is bounded because its support function

$$
x \in \mathbf{R}^{n} \longmapsto \Delta[x ; E(A)]:=\sup _{x \in E(A)}\langle z, x\rangle=[(x, A x\rangle]^{1 / 2}
$$

is finite. The ellipsoid $E(A)$ is centered at $0 \in \mathbf{R}^{n}$, but it may not contain 0 in its interior. Since there are matrices in $P_{n}$ which are singular, the possibility of having flat ellipsoids is not excluded.

Combinations of two or more ellipsoids appear quite often in applications. For instance, Hiriart-Urruty and Seeger [1, Corollary 6.2] obtained the bounds

$$
\bigcap_{i \in I(\bar{x})} E\left(\nabla^{2} f_{i}(\bar{x})\right) \subset \partial^{2} f(\bar{x}) \subset \operatorname{co}\left[\bigcup_{i \in I(\bar{x})} E\left(\nabla^{2} f_{i}(\bar{x})\right)\right]
$$

for the second-order subdifferential $\partial^{2} f(\bar{x})$ of a convex function $f$ of the type

$$
x \in \mathbf{R}^{n} \longmapsto f(x)=\operatorname{Max}_{1 \leqslant i \leqslant p}\left\{f_{i}(x)\right\}
$$

(Each $f_{i}: \mathbf{R}^{n} \rightarrow \mathbf{R}$ is assumed to be convex and twice differentiable at $\bar{x} \in \mathbf{R}^{n}$, and $I(\bar{x})=\left\{i: f(\bar{x})=f_{i}(\bar{x})\right\}$ denotes the set of indices which are active at $\bar{x}$.) As a

Received 4th December, 1991.

Copyright Clearance Centre, Inc. Serial-fee code: 0004-8729/92 \$A2.00+0.00. 
second example we mention that Kurzhanski and Valyi [2] were concerned with a state estimation problem for dynamic systems under uncertainty, and they needed to estimate the intersection and the Minkowski sum of a finite number of ellipsoids. Unfortunately, the estimates announced in [2] were given without proof.

The purpose of this note is to derive, in a unified way, formulas for the Minkowski sum, the convex hull, the intersection, and the inverse sum of a finite family of ellipsoids.

For convenience we recall first some basic definitions and results. Let $\left\{A_{1}, \ldots, A_{\mathbf{p}}\right\}$ be a finite collection of matrices in $P_{n}$. The series sum of the $A_{i}^{\prime} s$ is the ordinary sum $A_{1}+\cdots+A_{p} \in P_{n}$. The parallel sum of the $A_{i}^{\prime} s$ is the matrix $A_{1} \square \ldots \square A_{p} \in P_{n}$ defined by

$$
\left\langle x,\left[A_{1} \square \ldots \square A_{p}\right] x\right\rangle=\inf _{x_{1}+\cdots+x_{p}=x}\left\{\left\langle x_{1}, A_{1} x_{1}\right\rangle+\cdots+\left\langle x_{p}, A_{p} x_{p}\right\rangle\right\} \quad \text { for all } x \in \mathbf{R}^{n} .
$$

If the $A_{i}^{\prime} s$ are nonsingular, then it is possible to write the following pair of dual formulas:

$$
\begin{aligned}
A_{1} \square \ldots \square A_{p} & =\left(A_{1}^{-1}+\cdots+A_{p}^{-1}\right)^{-1}, \\
A_{1}+\cdots+A_{p} & =\left(A_{1}^{-1} \square \ldots \square A_{p}^{-1}\right)^{-1} .
\end{aligned}
$$

For a detailed discussion on the parallel addition of matrices, see for instance Mazure $[3,4]$ and references therein. The following result is taken from Seeger $[6$, Theorem 8.1].

Lemma 1.1. Let $\left\{A_{1}, \ldots, A_{p}\right\}$ be a finite collection of matrices in $P_{n}$. Then

$$
\begin{gathered}
E\left(A_{1}+\cdots+A_{p}\right)=\bigcup_{\lambda \in \Lambda}\left\{\sqrt{\lambda_{1}} E\left(A_{1}\right)+\cdots+\sqrt{\lambda_{p}} E\left(A_{p}\right)\right\}, \\
E\left(A_{1} \square \ldots \square A_{p}\right)=\bigcup_{\lambda \in \Lambda}\left\{\sqrt{\lambda_{1}} E\left(A_{1}\right) \cap \cdots \cap \sqrt{\lambda_{p}} E\left(A_{p}\right)\right\},
\end{gathered}
$$

where $\Lambda$ stands for the elementary simplex in $\mathbf{R}^{p}$ :

$$
\Lambda:=\left\{\lambda=\left(\lambda_{1}, \ldots, \lambda_{p}\right) \in \mathbf{R}^{p}: \lambda_{1}+\cdots+\lambda_{p}=1 \text { and } \lambda_{i} \geqslant 0 \text { for all } i=1, \ldots, p\right\} .
$$

The formulas given in Lemma 1.1 are crucial for the understanding of the theorems stated in the next section. For the sake of completeness in our exposition we need to record also the following result:

Lemma 1.2. Let $\left\{A_{1}, \ldots, A_{p}\right\}$ be a finite collection of matrices in $P_{n}$. Then the following equality is true

$$
E\left(A_{1} \square \ldots \square A_{p}\right)=\bigcap_{\substack{B_{1}, \ldots, B_{p} \\ B_{1}+\cdots+B_{p}=I_{n}}} E\left(B_{1} A_{1} B_{1}^{T}+\cdots+B_{p} A_{p} B_{p}^{T}\right) .
$$


Here $I_{n}$ denotes the $n \times n$ identity matrix, and $B_{1}^{T}, \ldots, B_{p}^{T}$ are the transposes of the $n \times n$ matrices $B_{1}, \ldots, B_{p}$, respectively.

PROOF: Let $x \in \mathbf{R}^{n}$ be arbitrary. If $x_{1}+\cdots+x_{p}=x$, then

$$
\left\langle x,\left[A_{1} \square \ldots \square A_{p}\right] x\right\rangle \leqslant\left\langle x_{1}, A_{1} x_{1}\right\rangle+\cdots+\left\langle x_{p}, A_{p} x_{p}\right\rangle .
$$

If one chooses $x_{i}=B_{i}^{T} x$, with $B_{1}+\cdots+B_{p}=I_{n}$, then one gets

$$
\left\langle x,\left[A_{1} \square \ldots \square A_{p}\right] x\right\rangle \leqslant\left\langle x,\left[B_{1} A_{1} B_{1}^{T}+\cdots+B_{p} A_{p} B_{p}^{T}\right] x\right\rangle,
$$

and thus

$$
E\left(A_{1} \square \ldots \square A_{p}\right) \subset E\left(B_{1} A_{1} B_{1}^{T}+\cdots+B_{p} A_{p} B_{p}^{T}\right)
$$

This proves of course the inclusion $E\left(A_{1} \square \ldots \square A_{p}\right) \subset K$, where $K$ is the set on the right hand side of (1.2). If the $A_{i}^{\prime} s$ are nonsingular, then one can show that the infimum in $(1.1)$ is attained at

$$
x_{i}=A_{i}^{-1}\left(A_{1} \square \ldots \square A_{p}\right) x \text { for all } i=1, \ldots, p .
$$

This implies that the inclusion (1.3) becomes an equality when

$$
B_{i}^{T}=A_{i}^{-1}\left(A_{1} \square \ldots \square A_{p}\right) \quad \text { for all } \quad i=1, \ldots, p .
$$

Summarising, formula (1.2) holds if the $A_{i}^{\prime} s$ are nonsingular. To cover the singular case one can use a continuity argument: one applies formula (1.2) to the collection of nonsingular matrices $\left\{A_{1}+\varepsilon I_{n}, \ldots, A_{p}+\varepsilon I_{n}\right\}$, where $\varepsilon>0$ is understood as a perturbation parameter. One gets in this way

$$
E\left(\left(A_{1}+\varepsilon I_{n}\right) \square \ldots \square\left(A_{p}+\varepsilon I_{n}\right)\right)
$$

Taking into account that

$$
E\left(B_{1}\left(A_{1}+\varepsilon I_{n}\right) B_{1}^{T}+\cdots+B_{p}\left(A_{p}+\varepsilon I_{n}\right) B_{p}^{T}\right) \supset E\left(B_{1} A_{1} B_{1}^{T}+\cdots+B_{p} A_{p} B_{p}^{T}\right),
$$

one obtains

$$
E\left(\left(A_{1}+\varepsilon I_{n}\right) \square \ldots \square\left(A_{p}+\varepsilon I_{n}\right)\right) \supset K \text {. }
$$

It suffices now to let $\varepsilon>0$ tend to zero in order to get the reverse inclusion $E\left(A_{1} \square \ldots \square A_{p}\right) \supset K$, and the proof is complete. 


\section{Calculus Rules for Combinations of Bounded Ellipsoids}

Let $\left\{A_{1}, \ldots, A_{p}\right\}$ be a finite collection of matrices in $P_{n}$. Our first rule concerns the convex hull of the ellipsoids $E\left(A_{1}\right), \ldots, E\left(A_{p}\right)$.

Theorem 2.1. (Calculus rule for the convex hull). The following equality is true:

$$
\operatorname{co}\left[\bigcup_{i=1}^{p} E\left(A_{i}\right)\right]=\bigcup_{\alpha \in \Lambda} E\left(\alpha_{1} A_{1}+\cdots+\alpha_{p} A_{p}\right) .
$$

Proof: Let $K$ be the set on the right hand side of (2.1). According to Lemma 1.1 one can write

$$
K=\bigcup_{\alpha \in \Lambda} \bigcup_{\lambda \in \Lambda}\left\{\sqrt{\lambda_{1}} E\left(\alpha_{1} A_{1}\right)+\cdots+\sqrt{\lambda_{p}} E\left(\alpha_{p} A_{p}\right)\right\}
$$

Taking into account that

$$
E\left(\alpha_{i} A_{i}\right)=\sqrt{\alpha_{i}} E\left(A_{i}\right) \text { for all } i=1, \ldots, p,
$$

one gets

$$
K=\bigcup_{\substack{\alpha \in \Lambda \\ \lambda \in \Lambda}}\left\{\sqrt{\lambda_{1}} \sqrt{\alpha_{1}} E\left(A_{1}\right)+\cdots+\sqrt{\lambda_{p}} \sqrt{\alpha_{p}} E\left(A_{p}\right)\right\}
$$

The coefficients $\alpha_{i}^{\prime} s$ and $\lambda_{i}^{\prime} s$ play the same role in the above union, so that one can impose on them the additional constraint

$$
\alpha_{i}=\lambda_{i} \quad \text { for all } i=1, \ldots, p
$$

One gets in this way

$$
K=\bigcup_{\alpha \in \Lambda}\left\{\alpha_{1} E\left(A_{1}\right)+\cdots+\alpha_{p} E\left(A_{p}\right)\right\}
$$

But this is precisely the convex hull of the sets $E\left(A_{1}\right), \ldots, E\left(A_{p}\right)$ (see Rockafellar $[5$, p.80]).

REMARK 2.1. The convex hull of the ellipsoids $E\left(A_{1}\right), \ldots, E\left(A_{p}\right)$ admits the alternative formula

$$
\operatorname{co}\left[\bigcup_{i=1}^{p} E\left(A_{i}\right)\right]=\overline{\bigcup_{\alpha \in \Lambda^{+}} E\left(\alpha_{1} A_{1}+\cdots+\alpha_{p} A_{p}\right)}
$$

where $\bar{C}$ stands for the closure of the set $C \subset \mathbb{R}^{n}$, and

$$
\Lambda^{+}:=\left\{\lambda=\left(\lambda_{1}, \ldots, \lambda_{p}\right) \in \mathbb{R}^{p}: \lambda_{1}+\cdots+\lambda_{p}=1, \quad \lambda_{i}>0 \quad \text { for all } \quad i=1, \ldots, p\right\}
$$


Formula (2.3) follows from Theorem 2.1 and the fact that the mapping $\lambda \in \Lambda \longmapsto$ $E\left(\lambda_{1} A_{1}+\cdots+\lambda_{p} A_{p}\right)$ is continuous with respect to the Hausdorff distance.

The next rule refers to the operation called inverse addition (see Rockafellar [5, p.21]). Recall that the inverse sum of the ellipsoids $E\left(A_{1}\right), \ldots, E\left(A_{p}\right)$ is the set

$$
E\left(A_{1}\right) \otimes \cdots \otimes E\left(A_{p}\right):=\bigcup_{\alpha \in \Lambda}\left\{\alpha_{1} E\left(A_{1}\right) \cap \cdots \cap \alpha_{p} E\left(A_{p}\right)\right\}
$$

THEOREM 2.2. (Calculus rule for the inverse sum). The following formula holds:

$$
E\left(A_{1}\right) \otimes \cdots \otimes E\left(A_{p}\right)=\bigcup_{\alpha \in \Lambda} E\left(\alpha_{1} A_{1} \square \ldots \square \alpha_{p} A_{p}\right) .
$$

Proof: The proof of equality (2.5) follows the same steps as in the previous theorem. This time one has to apply, of course, the second formula appearing in Lemma 1.1 .

REMARK 2.2. If $\alpha_{i}=0$ for some $i \in\{1, \ldots, p\}$, then the parallel sum $\alpha_{1} A_{1} \square \ldots \square \alpha_{p} A_{p}$ reduces to the zero matrix, and consequently

$$
E\left(\alpha_{1} A_{1} \square \ldots \square \alpha_{p} A_{p}\right)=\{0\} .
$$

This means that the union in (2.5) remains unchanged if one replaces $\Lambda$ by the smaller set $\Lambda^{+}$. Thus, one can also write

$$
E\left(A_{1}\right) \otimes \cdots \otimes E\left(A_{p}\right)=\bigcup_{\alpha \in \Lambda^{+}} E\left(\alpha_{1} A_{1} \square \ldots \square \alpha_{p} A_{p}\right) .
$$

We continue now with a rule for estimating the Minkowski sum of the ellipsoids $E\left(A_{1}\right), \ldots, E\left(A_{p}\right)$.

Theorem 2.3. (Calculus rule for the Minkowski sum). One has the formula

$$
E\left(A_{1}\right)+\cdots+E\left(A_{p}\right)=\bigcap_{\alpha \in \Lambda^{+}} E\left(\frac{1}{\alpha_{1}} A_{1}+\cdots+\frac{1}{\alpha_{p}} A_{p}\right) .
$$

Proof: Suppose first that the matrices $A_{1}, \ldots, A_{p}$ are non-singular. If one applies Theorem 2.2 to the collection $\left\{A_{1}^{-1}, \ldots, A_{p}^{-1}\right\}$, one gets

$$
E\left(A_{1}^{-1}\right) \otimes \cdots \otimes E\left(A_{p}^{-1}\right)=\bigcup_{\alpha \in \Lambda} E\left(\alpha_{1} A_{1}^{-1} \square \ldots \square \alpha_{p} A_{p}^{-1}\right),
$$

or equivalently,

$$
E\left(A_{1}^{-1}\right) \otimes \cdots \otimes E\left(A_{p}^{-1}\right)=\bigcup_{\alpha \in \Lambda^{+}} E\left(\alpha_{1} A_{1}^{-1} \square \ldots \square \alpha_{p} A_{p}^{-1}\right) .
$$


Now we compute the polar of each set appearing in the above equality. Recall that the polar $C^{0}$ of a set $C \subset \mathbb{R}^{n}$ is given by

$$
C^{0}:=\left\{z \in \mathbb{R}^{n}:\langle z, x\rangle \leqslant 1 \text { for all } x \in C\right\} .
$$

Applying standard rules for computing polar sets, one gets for the left hand side

$$
\left[E\left(A_{1}^{-1}\right) \otimes \cdots \otimes E\left(A_{p}^{-1}\right)\right]^{\circ}=\left[E\left(A_{1}^{-1}\right)\right]^{\circ}+\cdots+\left[E\left(A_{p}^{-1}\right)\right]^{0}=E\left(A_{1}\right)+\cdots+E\left(A_{p}\right),
$$

and for the right hand side

$$
\begin{aligned}
{\left[\bigcup_{\alpha \in \Lambda^{+}} E\left(\alpha_{1} A_{1}^{-1} \square \ldots \square \alpha_{p} A_{p}^{-1}\right)\right]^{\circ} } & =\bigcap_{\alpha \in \Lambda^{+}}\left[E\left(\alpha_{1} A_{1}^{-1} \square \ldots \square \alpha_{p} A_{p}^{-1}\right)\right]^{\circ} \\
& =\bigcap_{\alpha \in \Lambda^{+}} E\left(\left[\alpha_{1} A_{1}^{-1} \square \ldots \square \alpha_{p} A_{p}^{-1}\right]^{-1}\right) \\
& =\bigcap_{\alpha \in \Lambda^{+}} E\left(\frac{1}{\alpha_{1}} A_{1}+\cdots+\frac{1}{\alpha_{p}} A_{p}\right) .
\end{aligned}
$$

Summarising, we have proven that equality (2.7) holds when the matrices $A_{1}, \ldots, A_{p}$ are nonsingular. Consider now the singular case. Let $K$ be the set on the right hand side of (2.7). According to Lemma 1.1 one can write

$$
\begin{aligned}
K & =\bigcap_{\alpha \in \Lambda^{+}} \bigcup_{\lambda \in \Lambda}\left\{\sqrt{\lambda_{1}} E\left(\frac{1}{\alpha_{1}} A_{1}\right)+\cdots+\sqrt{\lambda_{p}} E\left(\frac{1}{\alpha_{p}} A_{p}\right)\right\} \\
& =\bigcap_{\alpha \in \Lambda^{+}} \bigcup_{\lambda \in \Lambda}\left\{\frac{\sqrt{\lambda_{1}}}{\sqrt{\alpha_{1}}} E\left(A_{1}\right)+\cdots+\frac{\sqrt{\lambda_{p}}}{\sqrt{\alpha_{p}}} E\left(A_{p}\right)\right\} .
\end{aligned}
$$

Since

$$
\bigcup_{\lambda \in \Lambda}\left\{\frac{\sqrt{\lambda_{1}}}{\sqrt{\alpha_{1}}} E\left(A_{1}\right)+\cdots+\frac{\sqrt{\lambda_{p}}}{\sqrt{\alpha_{p}}} E\left(A_{p}\right)\right\} \supset E\left(A_{1}\right)+\cdots+E\left(A_{p}\right)
$$

for all $\alpha \in \Lambda^{+}$, one has the trivial inclusion $K \supset E\left(A_{1}\right)+\cdots+E\left(A_{p}\right)$. To prove the reverse inclusion, we apply formula (2.7) to the nonsingular matrices $\left\{A_{1}+\varepsilon I_{n}, \ldots, A_{p}+\right.$ $\left.\varepsilon I_{n}\right\}$. In this way one gets

$$
E\left(A_{1}+\varepsilon I_{n}\right)+\cdots+E\left(A_{p}+\varepsilon I_{n}\right)=\bigcap_{\alpha \in \Lambda^{+}} E\left(\frac{1}{\alpha_{1}}\left(A_{1}+\varepsilon I_{n}\right)+\cdots+\frac{1}{\alpha_{p}}\left(A_{p}+\varepsilon I_{n}\right)\right) .
$$

But, for all $\alpha \in \Lambda^{+}$, one has

Hence

$$
E\left(\frac{1}{\alpha_{1}}\left(A_{1}+\varepsilon I_{n}\right)+\cdots+\frac{1}{\alpha_{p}}\left(A_{p}+\varepsilon I_{n}\right)\right) \supset E\left(\frac{1}{\alpha_{1}} A_{1}+\cdots+\frac{1}{\alpha_{p}} A_{p}\right) .
$$

$$
E\left(A_{1}+\varepsilon I_{n}\right)+\cdots+E\left(A_{p}+\varepsilon I_{n}\right) \supset K \text {. }
$$


To get the desired inclusion, it suffices now to let the parameter $\varepsilon>0$ tend to sero.

Formula (2.7) has been announced in a slightly different form by Kurzhanski and Vályi [2, Lemma 4.1]. These authors gave without proof the following result:

where

$$
\begin{gathered}
E\left(a_{1} ; A_{1}\right)+\cdots+E\left(a_{p} ; A_{p}\right)=\bigcap_{\pi_{i}>0} E\left(a_{1}+\cdots+a_{p} ; A(\pi)\right) \\
A(\pi):=\left(\pi_{1}+\cdots+\pi_{p}\right)\left(\frac{1}{\pi_{1}} A_{1}+\cdots+\frac{1}{\pi_{p}} A_{p}\right)
\end{gathered}
$$

The notation $E(a ; A)$ stands for the ellipsoid associated with $A \in P_{n}$ which is bounded and centered at $a \in \mathbf{R}^{n}$, that is,

$$
E(a ; A):=\left\{z \in \mathbb{R}^{n}:\langle z, x\rangle \leqslant\langle a, x\rangle+[\langle x, A x\rangle]^{1 / 2} \quad \text { for all } \quad x \in \mathbb{R}^{n}\right\}=a+E(A) \text {. }
$$

A simple exercise shows that formulas (2.7) and (2.8) are equivalent.

We state finally a rule for estimating the intersection of the ellipsoids $E\left(A_{1}\right), \ldots$, $E\left(A_{p}\right)$.

THEOREM 2.4. (Calculus rule for the intersection). The following formula holds:

$$
E\left(A_{1}\right) \cap \cdots \cap E\left(A_{p}\right)=\bigcap_{\alpha \in \Lambda^{+}} E\left(\frac{1}{\alpha_{1}} A_{1} \square \ldots \square \frac{1}{\alpha_{p}} A_{p}\right) .
$$

Proof: This theorem can be proven in the same way as the previous one. We consider first the case in which the matrices $A_{1}, \ldots, A_{p}$ are nonsingular. Theorem 2.1 applied to the collection $\left\{A_{1}^{-1}, \ldots, A_{p}^{-1}\right\}$ yields

$$
\operatorname{co}\left[\bigcup_{i=1}^{p} E\left(A_{i}^{-1}\right)\right]=\bigcup_{\alpha \in \Lambda} E\left(\alpha_{1} A_{1}^{-1}+\cdots+\alpha_{p} A_{p}^{-1}\right),
$$

or equivalently,

$$
\operatorname{co}\left[\bigcup_{i=1}^{p} E\left(A_{i}^{-1}\right)\right]=\overline{\bigcup_{\alpha \in \Lambda^{+}} E\left(\alpha_{1} A_{1}^{-1}+\cdots+\alpha_{p} A_{p}^{-1}\right)} .
$$

Now we use the technique of computing the corresponding polar sets. One has on the 
right hand side

$$
\left\{\operatorname{co}\left[\bigcup_{i=1}^{p} E\left(A_{i}^{-1}\right)\right]\right\}^{0}=\bigcap_{i=1}^{p}\left\{E\left(A_{i}^{-1}\right)\right\}^{0}=\bigcap_{i=1}^{p} E\left(A_{i}\right),
$$

and on the left hand side

$$
\begin{aligned}
\left\{\bigcup_{\alpha \in \Lambda^{+}} E\left(\alpha_{1} A_{1}^{-1}+\cdots+\alpha_{p} A_{p}^{-1}\right)\right\}^{0} & =\bigcap_{\alpha \in \Lambda^{+}}\left\{E\left(\alpha_{1} A_{1}^{-1}+\cdots+\alpha_{p} A_{p}^{-1}\right)\right\}^{0} \\
& =\bigcap_{\alpha \in \Lambda^{+}} E\left(\left[\alpha_{1} A_{1}^{-1}+\cdots+\alpha_{p} A_{p}^{-1}\right]^{-1}\right) \\
& =\bigcap_{\alpha \in \Lambda^{+}} E\left(\frac{1}{\alpha_{1}} A_{1} \square \ldots \square \frac{1}{\alpha_{p}} A_{p}\right) .
\end{aligned}
$$

Finally, we consider the singular case. Let $K$ be the set on the right hand side of (2.9). Lemma 1.1 yields

$$
\begin{aligned}
K & =\bigcap_{\alpha \in \Lambda+} \bigcup_{\lambda \in \Lambda}\left\{\sqrt{\lambda_{1}} E\left(\frac{1}{\alpha_{1}} A_{1}\right) \cap \cdots \cap \sqrt{\lambda_{p}} E\left(\frac{1}{\alpha_{p}} A_{p}\right)\right\} \\
& =\bigcap_{\alpha \in \Lambda^{+}} \bigcup_{\lambda \in \Lambda}\left\{\frac{\sqrt{\lambda_{1}}}{\sqrt{\alpha_{1}}} E\left(A_{1}\right) \cap \cdots \cap \frac{\sqrt{\lambda_{p}}}{\sqrt{\alpha_{p}}} E\left(A_{p}\right)\right\} .
\end{aligned}
$$

One then has the trivial inclusion $K \supset E\left(A_{1}\right) \cap \cdots \cap E\left(A_{p}\right)$. To prove the reverse inclusion, we apply formula (2.9) to the collection $\left\{A_{1}+\varepsilon I_{n}, \ldots, A_{p}+\varepsilon I_{n}\right\}$. In this way one gets

$$
E\left(A_{1}+\varepsilon I_{n}\right) \cap \cdots \cap E\left(A_{p}+\varepsilon I_{n}\right)=\bigcap_{\alpha \in \Lambda^{+}} E\left(\frac{1}{\alpha_{1}}\left(A_{1}+\varepsilon I_{n}\right) \square \ldots \square \frac{1}{\alpha_{p}}\left(A_{p}+\varepsilon I_{n}\right)\right) .
$$

But, for all $\alpha \in \Lambda^{+}$, one has

$$
E\left(\frac{1}{\alpha_{1}}\left(A_{1}+\varepsilon I_{n}\right) \square \ldots \square \frac{1}{\alpha_{p}}\left(A_{p}+\varepsilon I_{n}\right)\right) \supset E\left(\frac{1}{\alpha_{1}} A_{1} \square \ldots \square \frac{1}{\alpha_{p}} A_{p}\right) .
$$

\section{Hence}

$$
E\left(A_{1}+\varepsilon I_{n}\right) \cap \cdots \cap E\left(A_{p}+\varepsilon I_{n}\right) \supset K
$$

It suffices now to let $\varepsilon>0$ tend to zero.

Kurzhanski and Vályi [2, Lemma 4.2] gave a different estimate for the intersection of a finite family of ellipsoids. Their formula reads as follows:

$$
\bigcap_{i=1}^{p} E\left(a_{i} ; A_{i}\right)=\bigcap_{\substack{B_{1}, \ldots, B_{p} \\ B_{1}+\cdots+B_{p}=I_{n}}}\left\{E\left(B_{1} a_{1} ; B_{1} A_{1} B_{1}^{T}\right)+\cdots+E\left(B_{p} a_{p} ; B_{p} A_{p} B_{p}^{T}\right)\right\}
$$


If the ellipsoids are centered at the origin, then (2.10) takes the form

$$
\bigcap_{i=1}^{p} E\left(A_{i}\right)=\bigcap_{\substack{B_{1}, \ldots, B_{p} \\ B_{1}+\cdots+B_{p}=I_{n}}}\left\{E\left(B_{1} A_{1} B_{1}^{T}\right)+\cdots+E\left(B_{p} A_{p} B^{T}\right)\right\}
$$

Let us prove briefly that the complicated expression appearing on the right hand side of (2.11) reduces to the simpler estimate given in Theorem 2.4. If one applies Theorem 2.3 to the collection $\left\{B_{1} A_{1} B_{1}^{T}, \ldots, B_{p} A_{p} B_{p}^{T}\right\}$, one obtains

$$
\bigcap_{i=1}^{p} E\left(A_{i}\right)=\bigcap_{\substack{B_{1}, \ldots, B_{p} \\ B_{1}+\cdots+B_{p}=I_{n}}} \bigcap_{\alpha \in \Lambda^{+}} E\left(\frac{1}{\alpha_{1}} B_{1} A_{1} B_{1}^{T}+\cdots+\frac{1}{\alpha_{p}} B_{p} A_{p} B_{p}^{T}\right),
$$

or equivalently

$$
\bigcap_{i=1}^{p} E\left(A_{i}\right)=\bigcap_{\alpha \in \Lambda^{+}} \bigcap_{\substack{B_{1}, \ldots, B_{p} \\ B_{1}+\cdots+B_{p}=I_{n}}} E\left(B_{1}\left(\frac{1}{\alpha_{1}} A_{1}\right) B_{1}^{T}+\cdots+B_{p}\left(\frac{1}{\alpha_{p}} A_{p}\right) B_{p}^{T}\right) .
$$

It suffices now to apply Lemma 1.2 to obtain the formula given in Theorem 2.4.

\section{Application: Ellipsoidal Bounds for a Convex Polytope}

Theorems 2.1. and 2.3 can be used to construct inner and outer ellipsoidal approximations of a convex polytope which admits the representation

$$
L(R):=\{L x: x \in R\}
$$

Here $\quad L: \mathbb{R}^{n} \rightarrow \mathbb{R}^{n}$ is a linear mapping and $R$ stands for the bounded rectangular box

$$
R=\left[a_{1}, b_{1}\right] \times \cdots \times\left[a_{n}, b_{n}\right], \quad \text { with } a_{i} \leqslant b_{i} \quad \text { for all } i=1, \ldots, n \text {. }
$$

For notational convenience, $L$ is identified with the corresponding matrix relative to the canonical basis $\left\{e_{1}, \ldots, e_{n}\right\}$ of $\mathbf{R}^{n}$, and vectors of $\mathbf{R}^{n}$ are written in the column form. The rectangular box $R$ admits the expression $R=c_{R}+D_{R}$, where

$$
c_{R}:=\left(\frac{a_{1}+b_{1}}{2}, \ldots, \frac{a_{n}+b_{n}}{2}\right)^{T}
$$

corresponds to its centre, and

$$
D_{R}:=\left[-d_{1}, d_{1}\right] \times \cdots \times\left[-d_{n}, d_{n}\right], \quad \text { with } d_{i}:=\left(b_{i}-a_{i}\right) / 2 \text { for all } i=1, \ldots, n \text {, }
$$

is a translation of $R$ centered at the origin. The next proposition is a consequence of the fact that $D_{R}$ can be written as a Minkowski sum of ellipsoids. To see this, observe 
that the closed segment joining an arbitrary vector $v \in \mathbb{R}^{n}$ and its opposite $-v$ is a flat ellipsoid. Indeed, it is the ellipsoid associated with the rank one matrix $v v^{T}$, that is,

Thus

$$
\operatorname{co}\{v,-v\}=E\left(v v^{T}\right) \quad \text { for all } v \in \mathbf{R}^{n} .
$$

can be written in the form

$$
D_{R}=E\left(d_{1}^{2} e_{1} e_{1}^{T}\right)+\cdots+E\left(d_{n}^{2} e_{n} e_{n}^{T}\right) .
$$

To underline the number of ellipsoids involved in the representation of $D_{R}$, we use the notation

$\Lambda_{n}^{+}:=\left\{\lambda=\left(\lambda_{1}, \ldots, \lambda_{n}\right) \in \mathbb{R}^{n}: \lambda_{1}+\cdots+\lambda_{n}=1\right.$ and $\lambda_{i}>0$ for all $\left.i=1, \ldots, n\right\}$

Proposition 3.1. (Outer ellipsoidal approximation). Let $\ell_{i}=L e_{i}$ denote the $i^{\text {th }}$ column of $L$. Then, for each $\lambda \in \Lambda_{n}^{+}$, one has the upper bound

$$
L(R) \subset L c_{R}+E\left(\frac{d_{1}^{2}}{\lambda_{1}} \ell_{1} \ell_{1}^{T}+\cdots+\frac{d_{n}^{2}}{\lambda_{n}} \ell_{n} \ell_{n}^{T}\right) .
$$

If $L$ is invertible, one has more precisely

$$
L(R)=L c_{R}+\bigcap_{\lambda \in \Lambda_{n}^{+}} E\left(\frac{d_{1}^{2}}{\lambda_{1}} \ell_{1} \ell_{1}^{T}+\cdots+\frac{d_{n}^{2}}{\lambda_{n}} l_{n} \ell_{n}^{T}\right) .
$$

Proof: Theorem 2.3 applied to the Minkowski sum (3.1) yields

$$
D_{R}=\bigcap_{\lambda \in \Lambda_{n}^{+}} E\left(\frac{d_{1}^{2}}{\lambda_{1}} e_{1} e_{1}^{T}+\cdots+\frac{d_{1}^{2}}{\lambda_{1}} e_{n} e_{n}^{T}\right) .
$$

From this and the equality $L(R)=L c_{R}+L\left(D_{R}\right)$, one deduces that

$$
L(R) \subset L c_{R}+\bigcap_{\lambda \in \Lambda_{n}^{+}} L\left[E\left(\frac{d_{1}^{2}}{\lambda_{1}} e_{1} e_{1}^{T}+\cdots+\frac{d_{n}^{2}}{\lambda_{n}} e_{n} e_{n}^{T}\right)\right] .
$$

To prove the inclusion (3.2), it suffices now to apply the general formula

$$
L(E(A))=E\left(L A L^{T}\right) \quad \text { for all } \quad A \in P_{n} .
$$

If $L$ is invertible, then the inclusion (3.4) becomes an equality. 
To obtain an inner ellipsoidal approximation of $L(R)$, we write this time

$$
D_{R}=\operatorname{co}\left[\cup\left\{E\left(v v^{T}\right): v \text { is a vertex of } D_{R}\right\}\right]
$$

as a convex hull of ellipsoids. The $2^{n}$ vertices of $D_{R}$ are vectors of the form $\left( \pm d_{1}, \ldots, \pm d_{n}\right)^{T}$. Since $E\left(v v^{T}\right)=E\left((-v)(-v)^{T}\right)$, it suffices to take only half of them in the representation (3.5) (for instance, all the vertices with the sign + preceding the first component $d_{1}$ ). Theorem 2.1 yields in this case:

Proposition 3.2. (Inner ellipsoidal approximation). Let $\left\{v_{1}, \ldots, v_{p}\right\}$ be a collection of $p=2^{n-1}$ vertices of $D_{R}$. Suppose this collection does not contain two opposite vertices. Denote the elementary simplex in $R^{p}$ by $\Lambda_{p}$, and set $u_{i}:=L v_{i}$ for all $i=1, \ldots, p$.

Then

$$
L(R)=L c_{R}+\bigcup_{\lambda \in \Lambda_{p}} E\left(\lambda_{1} u_{1} u_{1}^{T}+\cdots+\lambda_{p} u_{p} u_{p}^{T}\right) .
$$

In particular, for each $\lambda \in \Lambda_{p}$, one has the inner bound

$$
L c_{R}+E\left(\lambda_{1} u_{1} u_{1}^{T}+\cdots+\lambda_{p} u_{p} u_{p}^{T}\right) \subset L(R) .
$$

Proof: Start by applying Theorem 2.1 to the convex hull

$$
D_{R}=\text { co }\left[\bigcup_{i=1}^{p} E\left(v_{i} v_{i}^{T}\right)\right] .
$$

The remaining part of the proof is similar to that of Proposition 3.1. The sole difference is that this time $L$ has to be permuted with a union symbol (instead of an intersection). This is always possible, even if $L$ is not invertible.

\section{REFERENCES}

[1] J.-B. Hiriart-Urruty and A. Seeger, 'Calculus rules on a new set-valued second-order derivative for convex functions', Nonlinear Anal.: Th. Meth and Appl. 13 (1989), 721-738.

[2] A.B. Kurshanski and I. Valyi, 'Elliproidal techniques : guaranteed atate estimation', Working paper, (International Institute for Applied Systems Analysis, Laxenburg, Austrie, 1991).

[3] M.L. Masure, Analyse variationnelle des formes quadratiques convexes, Thesis (University Paul Sabatier, Toulouse, 1986).

[4] M.L. Masure, 'L'addition parallele d'opérateurs interprétée comme inf-convolution de formes quadratiques convexes', Math. Modelling Numer.Anal. 20 (1986), 497-515.

[5] R.T. Rockafellar, Convex analysis (Princeton Univ.Press, New Jersey, 1970). 
[6] A. Seeger, 'Direct and inverse addition in convex analysis and applications', J. Math. Anal. Appl. 148 (1990), 317-349.

Université d'A vignon

Département de Mathématiques

84000 Avignon

France 